\title{
Characterizing the Mixed Volume
}

\author{
Vitali D. Milman and Rolf Schneider
}

\begin{abstract}
We investigate functional properties of the mixed volume that are sufficient to characterize it, up to elementary modifications.
\end{abstract}

2010 Mathematics Subject Classification: 52A39

\section{Introduction}

Let $\mathcal{K}^{n}$ denote the space of convex bodies (nonempty, compact, convex subsets) with the Hausdorff metric in $n$-dimensional Euclidean space $\mathbb{R}^{n}(n \geq 2)$ and $\mathcal{K}_{s}^{n}$ the subspace of centrally symmetric bodies. The mixed volume $V:\left(\mathcal{K}^{n}\right)^{n} \rightarrow \mathbb{R}$ is the unique symmetric function for which

$$
\left|\lambda_{1} K_{1}+\cdots+\lambda_{m} K_{m}\right|=\sum_{1 \leq i_{1}, \ldots, i_{n} \leq m} \lambda_{i_{1}} \cdots \lambda_{i_{n}} V\left(K_{i_{1}}, \ldots, K_{i_{n}}\right)
$$

for $m \in \mathbb{N}, K_{1}, \ldots, K_{m} \in \mathcal{K}^{n}, \lambda_{1}, \ldots, \lambda_{m} \geq 0$, where $|\cdot|$ denotes the volume. The non-trivial fact that $\left|\lambda_{1} K_{1}+\cdots+\lambda_{m} K_{m}\right|$ is indeed a polynomial, was emphasized by Minkowski. It is the basis of the Brunn-Minkowski theory of convex bodies, of which the mixed volume is a central notion; see [5], for example.

Motivated by an attempt to extend the mixed volume to log-concave measures, we have become interested in characterizing the classical mixed volume by some of its functional properties, not taking recourse to the notion of volume. Thus, we want to obtain the mixed volume directly as a multilinear function, instead of constructing it from the volume, using polynomial behaviour under Minkowski linear combinations.

The following properties of a function $F:\left(\mathcal{K}^{n}\right)^{n} \rightarrow \mathbb{R}$, which are shared by the mixed volume, are certainly good candidates for figuring in a characterization. Here a function $f: \mathcal{K}^{n} \rightarrow \mathbb{R}$ is called (Minkowski) additive if $f(K+L)=f(K)+f(L)$, where $K+L$ is the vector sum of $K, L \in \mathcal{K}^{n}$, and $f$ is increasing if $K \subset L$ implies $f(K) \leq f(L)$.

(P1) $F$ is Minkowski additive in each variable.

(P2) $F$ is increasing in each variable.

(P3) $F$ is symmetric (invariant under permutation of its arguments).

These important properties are far from being characteristic for the mixed volume, as shown by the following examples.

This research was supported by the Alexander von Humboldt Foundation. The first named author is also partially supported by ISF grant 387/09 and BSF grant no. 2006079. 
Example 1. Let $\mu$ be a finite, positive Borel measure on the group $\operatorname{GL}(n)$. By $h(K, \cdot)$ we denote the support function of the convex body $K$. For each $K \in \mathcal{K}^{n}$ we define a convex body $K^{\mu}$ by

$$
h\left(K^{\mu}, u\right)=\int_{\mathrm{GL}(n)} h(\alpha K, u) \mu(\mathrm{d} \alpha), \quad u \in \mathbb{R}^{n},
$$

(clearly this defines a support function) and put

$$
F\left(K_{1}, \ldots, K_{n}\right):=V\left(K_{1}^{\mu}, \ldots, K_{n}^{\mu}\right), \quad K_{1}, \ldots, K_{n} \in \mathcal{K}^{n} .
$$

Then $F$ has properties (P1), (P2), (P3). Moreover, $F$ is translation invariant in each variable.

Example 2. By $S^{n-1}$ we denote the unit sphere of $\mathbb{R}^{n}$. Let the function $f:\left(S^{n-1}\right)^{n} \rightarrow$ $\mathbb{R}$ be continuous, non-negative and even in each variable, and assume that $f$ is symmetric. Define

$$
\begin{aligned}
& F\left(K_{1}, \ldots, K_{n}\right) \\
& :=\int_{S^{n-1}} \ldots \int_{S^{n-1}} h\left(K_{1}, u_{1}\right) \cdots h\left(K_{n}, u_{n}\right) f\left(u_{1}, \ldots, u_{n}\right) \sigma\left(\mathrm{d} u_{1}\right) \cdots \sigma\left(\mathrm{d} u_{n}\right),
\end{aligned}
$$

where $\sigma$ is the spherical Lebesgue measure on $S^{n-1}$. If $t \in \mathbb{R}^{n}$ and $\langle\cdot, \cdot\rangle$ denotes the scalar product, then

$$
\begin{aligned}
& F\left(K_{1}+t, K_{2}, \ldots, K_{n}\right)-F\left(K_{1}, \ldots, K_{n}\right) \\
& =\int_{S^{n-1}} \cdots \int_{S^{n-1}}\left\langle u_{1}, t\right\rangle h\left(K_{2}, u_{2}\right) \cdots h\left(K_{n}, u_{n}\right) f\left(u_{1}, \ldots, u_{n}\right) \sigma\left(\mathrm{d} u_{1}\right) \cdots \sigma\left(\mathrm{d} u_{n}\right) \\
& =0
\end{aligned}
$$

since $f$ is even in $u_{1}$. Hence, $F$ is translation invariant in the first argument, and similarly in the other arguments. Therefore, we can always assume that $K_{i}$ contains the origin, for $i=1, \ldots, n$. Then it follows that $F$ is increasing in each argument. Thus, $F$ has properties (P1), (P2), (P3).

These functions $F$ are far from the mixed volume. In fact, the mixed volume even cannot be represented in the form

$$
V\left(K_{1}, \ldots, K_{n}\right)=\int_{\left(S^{n-1}\right)^{n}} h\left(K_{1}, u_{1}\right) \cdots h\left(K_{n}, u_{n}\right) \mu\left(\mathrm{d}\left(u_{1}, \ldots, u_{n}\right)\right),
$$

for all $K_{1}, \ldots, K_{n} \in \mathcal{K}^{n}$, with a finite signed Borel measure $\mu$ on $\left(S^{n-1}\right)^{n}$ (this follows from [5, Theorem 5.2.2]). We remark that, on the other hand, there exists a sequence $\left(f_{k}\right)_{k \in \mathbb{N}}$ of $C^{\infty}$ functions on $\left(S^{n-1}\right)^{n}$ with

$$
\int_{\left(S^{n-1}\right)^{n}} h\left(K_{1}, u_{1}\right) \cdots h\left(K_{n}, u_{n}\right) f_{k}\left(u_{1}, \ldots, u_{n}\right) \sigma^{n}\left(\mathrm{~d}\left(u_{1}, \ldots, u_{n}\right)\right) \rightarrow V\left(K_{1}, \ldots, K_{n}\right)
$$


as $k \rightarrow \infty$, for all $K_{1}, \ldots, K_{n} \in \mathcal{K}^{n}$. This was proved by Weil [8], and in a slightly sharper version $\left(f_{k}\right.$ real analytic) by Przesławski [4].

Example 3. Let $A_{2}, \ldots, A_{n} \in \mathcal{K}^{n}$ be fixed convex bodies and define

$$
F\left(K_{1}, \ldots, K_{n}\right):=\prod_{i=1}^{n} V\left(K_{i}, A_{2}, \ldots, A_{n}\right)
$$

for $K_{1}, \ldots, K_{n} \in \mathcal{K}^{n}$. Then $F$ has properties (P1), (P2), (P3).

If we demand only the properties (P1) and (P2), then each of the previous examples can be generalized in the obvious way. We give one further example with these properties.

Example 4. Embed $\mathbb{R}^{n}$ as a subspace in $\mathbb{R}^{N}$ with $N>n$ and let $\varphi_{1}, \ldots, \varphi_{n} \in \operatorname{GL}(n)$ be fixed linear maps from $\mathbb{R}^{n}$ into $\mathbb{R}^{N}$. Let $A_{n+1} \ldots, A_{N}$ be fixed convex bodies in $\mathbb{R}^{N}$ and define

$$
F\left(K_{1}, \ldots, K_{n}\right):=V\left(\varphi_{1} K_{1}, \ldots, \varphi_{n} K_{n}, A_{n+1}, \ldots, A_{N}\right)
$$

for $K_{1}, \ldots, K_{n} \in \mathcal{K}^{n}$, where $V$ denotes the mixed volume in $\mathbb{R}^{N}$. Then $F$ has properties $(\mathrm{P} 1)$ and $(\mathrm{P} 2)$.

These examples show clearly that, for a possible characterization of the mixed volume, further assumptions are necessary, which must somehow tie the variables of $F$ closer together. Here the criterion for the vanishing of the mixed volume comes to mind. It says (see [5, Th. 5.1.7]) that $V\left(K_{1}, \ldots, K_{n}\right)=0$ holds if and only if the $n$-tuple $\left(K_{1}, \ldots, K_{n}\right)$ is degenerate, in the sense of the following definition.

Definition. The $n$-tuple $\left(K_{1}, \ldots, K_{n}\right)$ of convex bodies in $\mathbb{R}^{n}$ is degenerate if there do not exist segments $S_{1} \subset K_{1}, \ldots, S_{n} \subset K_{n}$ with linearly independent directions.

Of the criterion for the vanishing of the mixed volume, we consider only the following two rudimentary forms.

(P4) $F\left(K_{1}, \ldots, K_{n}\right)=0$ if one of the arguments $K_{1}, \ldots, K_{n}$ is a singleton.

(P5) $F\left(K_{1}, \ldots, K_{n}\right)=0$ if two of the arguments $K_{1}, \ldots, K_{n}$ are parallel segments.

Properties (P1) and (P2) alone already have a number of implications, which we collect in the following lemma.

Lemma 1. Suppose that the function $F:\left(\mathcal{K}^{n}\right)^{n} \rightarrow \mathbb{R}$ satisfies $(\mathrm{P} 1)$ and $(\mathrm{P} 2)$. Then $F$ satisfies $(\mathrm{P} 4)$, and in each variable it is translation invariant, continuous and positively homogeneous. Moreover, $F \geq 0$.

If we restrict ourselves to centrally symmetric convex bodies, then conditions (P1), (P2) and (P5) turn out to be sufficient for a characterization of the mixed volume. It 
is a bit surprising that the seemingly weak condition (P5) for the vanishing of $F$ rules out so many other possibilities not leading to the mixed volume.

If we only consider $F$ on zonotopes, then even the monotonicity (P2) can be relaxed. (P2') $F\left(K, K_{2}, \ldots, K_{n}\right) \leq F\left(M, K_{2}, \ldots, K_{n}\right)$ whenever $K \subset M$ with $\operatorname{dim} K \leq 1$ and $\operatorname{dim} M \leq 2$; similarly in the other arguments.

By $\mathcal{Z}^{n}$ we denote the set of zonotopes, that is, of finite sums of segments, in $\mathbb{R}^{n}$.

Theorem 1. If the function $F:\left(\mathcal{Z}^{n}\right)^{n} \rightarrow \mathbb{R}$ satisfies $(\mathrm{P} 1),\left(\mathrm{P} 2^{\prime}\right),(\mathrm{P} 4)$ and $(\mathrm{P} 5)$, then

$$
F\left(K_{1}, \ldots, K_{n}\right)=a V\left(K_{1}, \ldots, K_{n}\right) \quad \text { for } K_{1}, \ldots, K_{n} \in \mathcal{Z}^{n},
$$

with a constant $a \geq 0$.

Under the full assumption of monotonicity, this result extends to centrally symmetric bodies and one non-symmetric body.

Theorem 2. If the function $F: \mathcal{K}^{n} \times\left(\mathcal{K}_{s}^{n}\right)^{n-1} \rightarrow \mathbb{R}$ satisfies (P1), (P2) and (P5), then

$$
F\left(K_{1}, \ldots, K_{n}\right)=a V\left(K_{1}, \ldots, K_{n}\right) \quad \text { for } K_{1} \in \mathcal{K}^{n} \text { and } K_{2}, \ldots, K_{n} \in \mathcal{K}_{s}^{n},
$$

with a constant $a \geq 0$.

An alternative formulation expresses a certain minimality property of the mixed volume among all functions satisfying properties (P1) and (P2).

Corollary. Let $F:\left(\mathcal{K}_{s}^{n}\right)^{n} \rightarrow \mathbb{R}$ satisfy $(\mathrm{P} 1)$ and $(\mathrm{P} 2)$. If $F$ is bounded from above by a constant multiple of the mixed volume, then $F$ is a constant multiple of the mixed volume.

In fact, under the assumptions of the Corollary, $F$ is non-negative, by Lemma 1. If there exists a constant $c$ with $0 \leq F\left(K_{1}, \ldots, K_{n}\right) \leq c V\left(K_{1}, \ldots, K_{n}\right)$ for all $K_{1}, \ldots, K_{n} \in \mathcal{K}_{s}^{n}$, then $F$ satisfies (P5), since $V$ does.

The case of non-symmetric bodies looks to be harder and seems to require different methods. The restriction to centrally symmetric convex bodies is clearly necessary in Theorem 2, since condition (P5) cannot distinguish between $K$ and $-K$. In fact, if $F\left(K_{1}, \ldots, K_{n}\right)$ is defined as a linear combination, with non-negative coefficients, of $V\left( \pm K_{1}, \ldots, \pm K_{n}\right)$, then $F$ satisfies (P1), (P2), (P5). One may conjecture that the converse is also true, but we have only been able to prove this in special cases and with additional assumptions. The next theorem settles the conjecture in the two-dimensional case. In the following, we write $-K=: K^{*}$.

Theorem 3. If the function $F:\left(\mathcal{K}^{2}\right)^{2} \rightarrow \mathbb{R}$ satisfies $(\mathrm{P} 1),(\mathrm{P} 2)$ and $(\mathrm{P} 5)$, then

$$
F(A, B)=a V\left(A, \alpha B+(1-\alpha) B^{*}\right)
$$

for $A, B \in \mathcal{K}^{2}$, with constants $a \geq 0$ and $\alpha \in[0,1]$. 
To extend this result (with two not necessarily symmetric arguments) to higher dimensions, we need an additonal condition, which expresses a further important property of the mixed volume.

(P6) $F\left(\varphi K_{1}, \ldots, \varphi K_{n}\right)=F\left(K_{1}, \ldots, K_{n}\right)$ for all $\varphi \in \mathrm{SL}(n)$ (whenever both sides of the equation are defined).

Theorem 4. If the function $F:\left(\mathcal{K}^{n}\right)^{2} \times\left(\mathcal{K}_{s}^{n}\right)^{n-2} \rightarrow \mathbb{R}$ satisfies (P1), (P2), (P5) and (P6), then

$$
F\left(A, B, C_{1}, \ldots, C_{n-2}\right)=a V\left(A, \alpha B+(1-\alpha) B^{*}, C_{1}, \ldots, C_{n-2}\right)
$$

for $A, B \in \mathcal{K}^{n}$ and $C_{1}, \ldots, C_{n-2} \in \mathcal{K}_{s}^{n}$, with constants $a \geq 0$ and $\alpha \in[0,1]$.

In view of the fact that the essential properties (P1) and (P2) are preserved under linear maps, it appears natural to ask whether the function defined by

$$
F\left(K_{1}, \ldots, K_{n}\right):=V\left(\varphi_{1} K_{1}, \ldots, \varphi_{n} K_{n}\right), \quad K_{1}, \ldots, K_{n} \in \mathcal{K}^{n},
$$

where $\varphi_{1}, \ldots, \varphi_{n} \in \mathrm{GL}(n)$ are fixed linear transformations, can be characterized by suitable properties. This function $F$ has in general none of the properties (P3), (P5), (P6). Property (P5) should be replaced by the following one.

(P5') To any nondegenerate segment $S$ there exists a nondegenerate segment $\bar{S}$, with unique direction, such that $F\left(S, \bar{S}, K_{3}, \ldots, K_{n}\right)=0$ (for all $K_{3}, \ldots, K_{n}$ such that the left side is defined), similarly for any other ordered pair of arguments.

What we can prove, is a corresponding generalization of Theorem 3 .

Theorem 5. If the function $F:\left(\mathcal{K}^{2}\right)^{2} \rightarrow \mathbb{R}$ satisfies $(\mathrm{P} 1),(\mathrm{P} 2)$ and $\left(\mathrm{P} 5^{\prime}\right)$, then

$$
F(A, B)=V\left(A, \varphi\left(\alpha B+(1-\alpha) B^{*}\right)\right)
$$

for $A, B \in \mathcal{K}^{2}$, with a linear transformation $\varphi \in \mathrm{GL}(2)$ and a constant $\alpha \in[0,1]$.

We prove Theorems 1 and 2 in the next section and Theorems 3 and 4 in Section 3. The proof of Theorem 5 follows separately in Section 4, since the construction of the linear map $\varphi$ requires an extra argument.

\section{Proof of Theorems 1 and 2}

In this section, we prove Theorem 1, Lemma 1, and Theorem 2.

Let $F$ be a function on $D(F)$ satisfying $(\mathrm{P} 1),\left(\mathrm{P} 2^{\prime}\right),(\mathrm{P} 4),(\mathrm{P} 5)$, where $D(F)$ is one of the domains of $F$ in Theorems 1 to 4 . If $F \equiv 0$, the assertions of Theorems 1 to 4 hold with $a=0$; therefore, we assume in the following that $F$ is not identically zero.

We prepare the proofs of Theorems 1 and 2 with first conclusions from the properties. 
Let $K_{2}, \ldots, K_{n}$ be fixed convex bodies for which $\left(S, K_{2}, \ldots, K_{n}\right) \in D(F)$ for segments $S$. For segments $S$ contained in a fixed line through the origin, the function $S \mapsto F\left(S, K_{2}, \ldots, K_{n}\right)$ is Minkowski additive, increasing and translation invariant. It follows that $F\left(S, K_{2}, \ldots, K_{n}\right)=f(\operatorname{length}(S))$, where $f$ is an increasing solution of Cauchy's functional equation and thus a linear function (e.g., see [1]). Thus, $F$ is positively homogeneous on segments, in each argument (observe that $F$ is translation invariant in each argument, by (P1) and (P4)).

All segments appearing in the following are assumed to have positive length. A segment is called centred if it has its centre at the origin $o$.

Proposition 1. Let $S, T_{1}, T_{2}$ be centred segments. If

$$
T_{1}+\mathbb{R} S=T_{2}+\mathbb{R} S
$$

then

$$
F\left(T_{1}, S, A_{1}, \ldots, A_{n-2}\right)=F\left(T_{2}, S, A_{1}, \ldots, A_{n-2}\right)
$$

for all convex bodies $A_{1}, \ldots, A_{n-2}$ for which $\left(S_{1}, S_{2}, A_{1}, \ldots, A_{n-2}\right) \in D(F)$ for segments $S_{1}, S_{2}$.

Here, the role of the first two arguments can be played by any other two arguments (in any order).

Proof. Under the assumption (1), we can choose $\lambda>0$ with $T_{1} \subset T_{2}+\lambda S$. Properties $\left(\mathrm{P} 2^{\prime}\right),(\mathrm{P} 1),(\mathrm{P} 5)$, applied in this order, give

$$
\begin{aligned}
& F\left(T_{1}, S, A_{1}, \ldots, A_{n-2}\right) \\
& \leq F\left(T_{2}+\lambda S, S, A_{1}, \ldots A_{n-2}\right) \\
& =F\left(T_{2}, S, A_{1}, \ldots, A_{n-2}\right)+F\left(\lambda S, S, A_{1}, \ldots, A_{n-2}\right) \\
& =F\left(T_{2}, S, A_{1}, \ldots, A_{n-2}\right) .
\end{aligned}
$$

Since the assumption is symmetric in $T_{1}$ and $T_{2}$, the assertion follows.

Proposition 2. $F$ is symmetric on segments.

Proof. Since $F$ is, in each variable, translation invariant and positively homogeneous on segments, we may restrict ourselves to centred segments of unit length. First let $n=2$, and let $S, T \subset \mathbb{R}^{2}$ be two centred unit segments. If $S$ and $T$ are parallel, then $0=F(S, T)=F(T, S)$, by (P5). Assume that $S, T$ have different directions. Let $D$ be a diagonal of the parallelogram $S+T$. Proposition 1 shows that $F(D, S)=F(T, S)$ and $F(D, T)=F(S, T)$. Also by Proposition $1, F(D, S)=F(D, T)$, hence $F(T, S)=$ $F(S, T)$.

This symmetry extends to $n$ dimensions. In $\mathbb{R}^{n}$, assume that the centred segments $S$ and $T$ have different directions. Let $\operatorname{span}\{S, T\}$ be the two-dimensional linear subspace spanned by $S$ and $T$. In the following, it is assumed that $\left(A, B, K_{3}, \ldots, K_{n}\right) \in D(F)$. 
The function $f$ defined by $f(A, B):=F\left(A, B, K_{3}, \ldots, K_{n}\right)$ (with fixed $K_{3}, \ldots, K_{n}$ ) for convex bodies $A, B$ in $\operatorname{span}\{S, T\}$ has properties (P1), (P2'), (P4), (P5) with respect to $\operatorname{span}\{S, T\}$. As just proved, it follows that $f(S, T)=f(T, S)$, which gives

$$
F\left(S, T, K_{3}, \ldots, K_{n}\right)=F\left(T, S, K_{3}, \ldots, K_{n}\right) .
$$

Of course, a similar symmetry holds for any other pair of arguments of $F$, and the symmetry of $F$ on segments follows.

Proof of Theorem 1. Under the assumptions of Theorem 1, we show that there is a constant $a \geq 0$ such that

$$
F\left(S_{1}, \ldots, S_{n}\right)=a V\left(S_{1}, \ldots, S_{n}\right)
$$

for all segments $S_{1}, \ldots, S_{n}$.

In order to give an inductive proof starting at dimension one, we extend the assertion to $n=1$, claiming that a function $F$ on closed intervals in $\mathbb{R}$ that is Minkowski additive, increasing and is zero on singletons, must be a constant multiple of the length of the intervals. This is true, as mentioned above.

Now let $n \geq 2$ and suppose that the assertion (3) has been proved in spaces of smaller dimension. Let $S, T_{2}, \ldots, T_{n}$ be segments, without loss of generality centred. We define

$$
f\left(K_{2}, \ldots, K_{n}\right):=F\left(S, K_{2}, \ldots, K_{n}\right)
$$

for $K_{2}, \ldots, K_{n} \in \mathcal{Z}^{n}$ with $K_{i} \subset S^{\perp}$, where $S^{\perp}$ denotes the $(n-1)$-dimensional linear subspace orthogonal to $S$. Then $f$ has properties (P1), (P2'), (P4), (P5), in the $(n-1)$ dimensional space $S^{\perp}$. Denoting by $V^{\prime}$ the mixed volume in $S^{\perp}$, we conclude from the induction hypothesis that there is a constant $c(S)$ such that

$$
F\left(S, S_{2}, \ldots, S_{n}\right)=c(S) V^{\prime}\left(S_{2}, \ldots, S_{n}\right)
$$

for all segments $S_{2}, \ldots, S_{n} \subset S^{\perp}$. Putting $a(S):=n c(S) / \operatorname{length}(S)$, we get from $[5$, (5.3.23)] that

$$
F\left(S, S_{2}, \ldots, S_{n}\right)=a(S) V\left(S, S_{2}, \ldots, S_{n}\right) .
$$

Since $F$ and $V$ are positively homogeneous on segments, we have $a(\lambda S)=a(S)$ for $\lambda>0$.

Let $S_{2}$ be the image of $T_{2}$ under orthogonal projection to $S^{\perp}$. From Proposition 1 we get

$$
F\left(S, T_{2}, T_{3}, \ldots, T_{n}\right)=F\left(S, S_{2}, T_{3}, \ldots, T_{n}\right),
$$

also if $S_{2}$ is one-pointed, since then $T_{2}$ is parallel to $S$, and both sides are zero. The last $n-2$ arguments can be treated similarly, hence

$$
F\left(S, T_{2}, \ldots, T_{n}\right)=F\left(S, S_{2}, \ldots, S_{n}\right)=a(S) V\left(S, S_{2}, \ldots, S_{n}\right)
$$

The latter is equal to $a(S) V\left(S, T_{2}, \ldots, T_{n}\right)$, since $V$ has the same properties as $F$. The obtained equality

$$
F\left(S, T_{2}, \ldots, T_{n}\right)=a(S) V\left(S, T_{2}, \ldots, T_{n}\right)
$$


holds for arbitrary $n$-tuples $S, T_{2}, \ldots, T_{n}$ of segments.

From Proposition 2 we have

$$
F\left(S, T_{2}, \ldots, T_{n}\right)=F\left(T_{2}, S, T_{3}, \ldots, T_{n}\right)=a\left(T_{2}\right) V\left(T_{2}, S, T_{3} \ldots, T_{n}\right),
$$

and we can conclude that $a(S)$ does not depend on $S$. This proves (3).

Using (P1), we can replace each line segment in (3) by a zonotope. This completes the proof of Theorem 1 .

Proof of Lemma 1. We prove a slightly more general version of Lemma 1, assuming that $F$ is a function on $D(F)$, where $D(F)$ is one of the domains of $F$ in Theorems 1 to 5 , and that $F$ satisfies (P1) and (P2) on this domain.

In the following it is assumed that $K, M, M^{\prime}, K_{3}, \ldots, K_{n}$ are convex bodies such that $\left(K, M, K_{3}, \ldots, K_{n}\right),\left(K, M^{\prime}, K_{3}, \ldots, K_{n}\right) \in D(F)$. For fixed $K_{3}, \ldots, K_{n}$, write $f(K, M):=F\left(K, M, K_{3}, \ldots, K_{n}\right)$. Below, $o$ is the origin of $\mathbb{R}^{n}, x \in \mathbb{R}^{n}$, and a singleton $\{x\}$ is written as $x$. From $f(o, M)=f(o+o, M)=2 f(o, M)$ we get $f(o, M)=0$.

Suppose there exist $x \in \mathbb{R}^{n}$ and and a convex body $M$ such that $f(x, M) \neq 0$. Since $0=f(o, M)=f(x-x, M)=f(x, M)+f(-x, M)$, we can assume (interchanging the roles of $x$ and $-x$, if necessary) that $f(x, M)>0$. We choose a convex body $M^{\prime}$ with $M \subset M^{\prime}$ and $o \subset M^{\prime}$; then $a:=f\left(x, M^{\prime}\right) \geq f(x, M)>0$. From $0=f\left(o, M^{\prime}\right)=$ $f\left(x, M^{\prime}\right)+f\left(-x, M^{\prime}\right)$ we get $f\left(-x, M^{\prime}\right)=-a$. Since $o \in M^{\prime}$, we have $M^{\prime} \subset 2 M^{\prime}$ and hence $-a=f\left(-x, M^{\prime}\right) \leq f\left(-x, 2 M^{\prime}\right)=2 f\left(-x, M^{\prime}\right)=-2 a$, a contradiction. This shows that $f(x, M)=0$ for all $x$ and $M$ and hence, by similar reasoning for the other arguments, that $F\left(K_{1}, \ldots, K_{n}\right)=0$ whenever $\left(K_{1}, \ldots, K_{n}\right) \in D(F)$ and one of the arguments is a singleton. Thus, $F$ satisfies $(\mathrm{P} 4)$. Together with (P2) this shows that $F \geq 0$.

From (P4) we get, in particular, that $F\left(K_{1}+x, K_{2}, \ldots, K_{n}\right)=F\left(K_{1}, K_{2}, \ldots, K_{n}\right)+$ $F\left(x, K_{2}, \ldots, K_{n}\right)=F\left(K_{1}, K_{2}, \ldots, K_{n}\right)$ for $\left(K_{1}, \ldots, K_{n}\right) \in D(F)$, and similarly in the other arguments; hence $F$ is translation invariant in each variable.

From (P1), (P2), (P4) we can deduce that the function $F$ is continuous in each variable; the proof is carried out in [2]. Additivity and continuity now also give positive homogeneity in each variable.

Now we assume that $F$ is a function on the domain as in Theorem 2 and that it satisfies (P1), (P2), (P5). Proposition 1 can be extended as follows.

Proposition 3. Let $k \in\{1, \ldots, n-1\}$, and let $S_{1}, \ldots, S_{k}$ be centred segments. If $K, M \in \mathcal{K}^{n}$ are convex bodies with

$$
K+\mathbb{R}\left(S_{1}+\cdots+S_{k}\right)=M+\mathbb{R}\left(S_{1}+\cdots+S_{k}\right),
$$

then

$$
F\left(K, S_{1}, \ldots, S_{k}, A_{1}, \ldots, A_{n-k-1}\right)=F\left(M, S_{1}, \ldots, S_{k}, A_{1}, \ldots, A_{n-k-1}\right)
$$

for $A_{1}, \ldots, A_{n-k-1} \in \mathcal{K}_{s}^{n}$. 
Proof. Under the assumption (4), we can choose $\lambda>0$ with $K \subset M+\lambda\left(S_{1}+\cdots+S_{k}\right)$. Properties (P2), (P1), (P5), applied in this order, give

$$
\begin{aligned}
& F\left(K, S_{1}, \ldots, S_{k}, A_{1}, \ldots, A_{n-k-1}\right) \\
& \leq F\left(M+\lambda\left(S_{1}+\cdots+S_{k}\right), S_{1}, \ldots, S_{k}, A_{1}, \ldots, A_{n-k-1}\right) \\
& =F\left(M, S_{1}, \ldots, S_{k}, A_{1}, \ldots, A_{n-k-1}\right)+\sum_{i=1}^{k} F\left(\lambda S_{i}, S_{1}, \ldots, S_{k}, A_{1}, \ldots, A_{n-k-1}\right) \\
& =F\left(M, S_{1}, \ldots, S_{k}, A_{1}, \ldots, A_{n-k-1}\right) .
\end{aligned}
$$

Since the assumption is symmetric in $K$ and $M$, the assertion follows.

Proof of Theorem 2. Under the assumptions of Theorem 2, $F$ has all the properties stated in Lemma 1. It follows from Theorem 1 that

$$
F\left(Z, S_{2}, \ldots, S_{n}\right)=a V\left(Z, S_{2}, \ldots, S_{n}\right)
$$

with a constant $a$, for zonotopes $Z$ and segments $S_{2}, \ldots, S_{n}$. By continuity, this equation holds also if $Z$ is a zonoid. We use an argument that we have learned from [3]. Let $K$ be a generalized zonoid, that is, a convex body for which there exist zonoids $Z_{1}, Z_{2}$ such that $K+Z_{1}=Z_{2}$. Then we get

$$
\begin{aligned}
& F\left(K, S_{2}, \ldots, S_{n}\right)+F\left(Z_{1}, S_{2}, \ldots, S_{n}\right)=F\left(K+Z_{1}, S_{2}, \ldots, S_{n}\right) \\
& =F\left(Z_{2}, S_{2}, \ldots, S_{n}\right)=a V\left(Z_{2}, S_{2}, \ldots, S_{n}\right)=a V\left(K+Z_{1}, S_{2}, \ldots, S_{n}\right) \\
& =a V\left(K, S_{2}, \ldots, S_{n}\right)+a V\left(Z_{1}, S_{2}, \ldots, S_{n}\right) \\
& =a V\left(K, S_{2}, \ldots, S_{n}\right)+F\left(Z_{1}, S_{2}, \ldots, S_{n}\right)
\end{aligned}
$$

and hence

$$
F\left(K, S_{2}, \ldots, S_{n}\right)=a V\left(K, S_{2}, \ldots, S_{n}\right) .
$$

Every centrally symmetric convex body with a support function of class $C^{\infty}$ is a generalized zonoid (see, e.g., [5, Section 3.5], in particular Theorem 3.5.3). Therefore, the generalized zonoids are dense in the set of all centrally symmetric convex bodies. Since $F$ and $V$ are continuous, the last equality holds for all centrally symmetric convex bodies $K$. In the same way, each of the last $n-1$ arguments can be replaced by a centrally symmetric convex body.

It remains to replace one of the convex bodies by a non-symmetric body.

Let $K \in \mathcal{K}^{n}$, and let $S_{2}, \ldots, S_{n}$ be segments with linearly independent directions. It follows from Proposition 3 and the translation invariance of $F$ in its first variable that

$$
F\left(K, S_{2}, \ldots, S_{n}\right)=F\left(K^{*}, S_{2}, \ldots, S_{n}\right) .
$$


Now the already proved part of Theorem 2 for centrally symmetric bodies gives

$$
\begin{aligned}
2 F\left(K, S_{2}, \ldots, S_{n}\right) & =F\left(K+K^{*}, S_{2}, \ldots, S_{n}\right) \\
& =a V\left(K+K^{*}, S_{2}, \ldots, S_{n}\right)=2 a V\left(K, S_{2}, \ldots, S_{n}\right) .
\end{aligned}
$$

As above, this can be extended to

$$
F\left(K, C_{2}, \ldots, C_{n}\right)=a V\left(K, C_{2}, \ldots, C_{n}\right) \quad \text { for } C_{2}, \ldots, C_{n} \in \mathcal{K}_{s}^{n} .
$$

This completes the proof of Theorem 2 .

\section{Proof of Theorems 3 and 4}

Already the assertions of the remaining theorems indicate that the proofs must be of an essentially different kind: a Minkowski combination $\alpha B+(1-\alpha) B^{*}$ appears as an argument, and the number $\alpha$ has to be found. For the treatment of centrally symmetric bodies in the previous section, the case of segments was crucial. This cannot be sufficient now. However, for non-symmetric bodies, a similar role can be played by the triangles (see the end of the proof of Theorem 4). For this reason, much of the following proof employs two-dimensional convex bodies. The additivity property must now be exploited in a deeper way: we use a result of Firey [2], which is based on a version of the Riesz representation theorem. With this, we construct an additive map on two-dimensional convex bodies and then find that it is essentially of the form $B \mapsto \alpha B+(1-\alpha) B^{*}$ (see Lemma 3 below); this is the crucial tool of the proofs.

Let $F$ satisfy the assumptions of Theorem 4, but without (P6) for a while, and let $F \not \equiv 0$. As shown in Section $2, F$ is then continuous and positively homogeneous in each argument, non-negative, and symmetric on segments. Further, we show that $F$ satisfies the following condition (P7).

(P7) If $F\left(K_{1}, \ldots, K_{n}\right)=0$, then $\left(K_{1}, \ldots, K_{n}\right)$ is degenerate.

For the proof, suppose that (P7) were false. Then there is an $n$-tuple $\left(K_{1}^{0}, \ldots, K_{n}^{0}\right)$ with $F\left(K_{1}^{0}, \ldots, K_{n}^{0}\right)=0$ which is not degenerate, hence there are segments $S_{1} \subset$ $K_{1}^{0}, \ldots, S_{n} \subset K_{n}^{0}$ with linearly independent directions. Since $F$ is increasing in each variable, it follows that $F\left(S_{1}, \ldots, S_{n}\right)=0$. We put $C:=S_{1}+\cdots+S_{n}$ and assert that

$$
F(\underbrace{C, \ldots, C}_{k}, S_{r_{k+1}}, \ldots, S_{r_{n}})=0
$$

for $k \in\{0, \ldots, n\}$ and $r_{k+1}, \ldots, r_{n} \in\{1, \ldots, n\}$. We prove this by induction on $k$. The case $k=0$ holds by assumption and by the symmetry of $F$ on segments if $r_{1}, \ldots, r_{n}$ are all different, and by (P5) if they are not all different. Suppose (5) holds for some $k \in\{0, \ldots, n-1\}$. Adding over $r_{k+1}$ from 1 to $n$, we obtain

$$
F(\underbrace{C, \ldots, C}_{k+1}, S_{r_{k+2}}, \ldots, S_{r_{n}})=0
$$


which completes the induction.

For given $K_{1}, \ldots, K_{n} \in \mathcal{K}^{n}$ we can choose $S_{1}, \ldots, S_{n}$ in such a way (by homogeneity and translation invariance) that $K_{1}, \ldots, K_{n} \subset C$. Then the case $k=n$ of (5) shows that $F\left(K_{1}, \ldots, K_{n}\right)=0$, a contradiction to $F \not \equiv 0$. This shows that $F$ satisfies (P7).

We fix $n-2$ centred segments $U_{1}, \ldots, U_{n-2}$ with linearly independent directions and also a two-dimensional linear subspace $E$ that is complementary to $\operatorname{span}\left\{U_{1}, \ldots, U_{n-2}\right\}$, the linear subspace spanned by $U_{1}, \ldots, U_{n-2}$ (with $E=\mathbb{R}^{2}$ if $n=2$ ). We denote by $\mathcal{K}(E)$ the set of convex bodies in $E$ and by $\mathcal{K}_{(0)}(E)$ the subset of bodies with nonempty interior relative to $E$. Let $B \in \mathcal{K}_{(0)}(E)$.

The function defined by $A \mapsto F\left(A, B, U_{1}, \ldots, U_{n-2}\right), A \in \mathcal{K}^{n}$, is not identically zero by (P7), it is Minkowski additive, positively homogeneous, increasing, and zero on singletons. These are the assumptions under which Firey's work [2] yields the following result, which we formulate here as a lemma.

Lemma 2. Under the previous assumptions, there are a number $p \in\{1, \ldots, n\}$ and $n-p$ pairwise orthogonal (centred) unit segments $\sigma_{1}, \ldots, \sigma_{n-p}$ such that one of the following cases occurs.

Case 1. $p \geq 2$, and there is a convex body $K$ of dimension $p$, contained in the orthogonal complement of $\operatorname{span}\left\{\sigma_{1}, \ldots, \sigma_{n-p}\right\}$, such that

$$
F\left(A, B, U_{1}, \ldots, U_{n-2}\right)=V(A, \underbrace{K, \ldots, K}_{p-1}, \sigma_{1}, \ldots, \sigma_{n-p})
$$

for all $A \in \mathcal{K}^{n}$. The body $K$ is uniquely determined up to a translation.

Case 2. $p=1$, and there is a constant $c>0$ such that

$$
F\left(A, B, U_{1}, \ldots, U_{n-2}\right)=c V\left(A, \sigma_{1}, \ldots, \sigma_{n-1}\right)
$$

for all $A \in \mathcal{K}^{n}$.

We assume in the following, without loss of generality, that $K$ has its Steiner point at the origin $o$; it is then uniquely determined.

A comment is in order which concerns the application of Minkowski's existence theorem in [2]. Firey's argument yields the existence of a finite Borel measure $\mu$ (depending on $U_{1}, \ldots, U_{n-2}$ and $B$ ) on $S^{n-1}$ with

$$
F\left(A, B, U_{1}, \ldots, U_{n-2}\right)=\frac{1}{n} \int_{S^{n-1}} h(A, u) \mu(\mathrm{d} u) \quad \text { for all } A \in \mathcal{K}^{n} .
$$

The number $p$ appearing in Case 1 is the dimension of the smallest linear subspace $L \subset \mathbb{R}^{n}$ containing the support of $\mu$, and the convex body $K$ is obtained by applying Minkowski's existence theorem in $L$ to the measure $\mu$, considered as a measure on $S^{n-1} \cap L$. This theorem does not hold in a one-dimensional space. Therefore, Firey's equation (5) is not valid for $p=1$ : if $\omega^{\prime}$ (in Firey's notation) contains precisely one point of the 0 -sphere $\Omega^{\prime}=S^{n-1} \cap L$, then $\mu\left(\omega^{\prime}\right)=c$ can be any positive number, whereas $s\left(\bar{K} ; \omega^{\prime}\right)=1$. This is the reason for the appearance of the constant $c$ (which is missing in [2]) in Case 2. 
Now we apply Lemma 2. Suppose, first, that Case 2 holds. Then we have

$$
F\left(A, B, U_{1}, \ldots, U_{n-2}\right)=c V\left(A, \sigma_{1}, \ldots, \sigma_{n-1}\right)=0
$$

for all convex bodies $A \subset \operatorname{span}\left\{\sigma_{1}, \ldots, \sigma_{n-1}\right\}$. We can choose a segment $S_{1} \subset$ $\operatorname{span}\left\{\sigma_{1}, \ldots, \sigma_{n-1}\right\}$ with $\operatorname{dim} \operatorname{span}\left\{U_{1}, \ldots, U_{n-2}, S_{1}\right\}=n-1$ and then (since $\operatorname{dim} B=$ $2)$ a segment $S_{2} \subset B$ with $\operatorname{dim} \operatorname{span}\left\{U_{1}, \ldots, U_{n-2}, S_{1}, S_{2}\right\}=n$. The $n$-tuple $\left(U_{1}, \ldots, U_{n-2}, S_{1}, S_{2}\right)$ is not degenerate, hence $F\left(S_{1}, B, U_{1}, \ldots, U_{n-2}\right) \neq 0$ by (P7). This is a contradiction, hence Case 2 does not occur.

In Case $1, p \geq 2$, and by (P5) we have

$$
V(U_{i}, \underbrace{K, \ldots, K}_{p-1}, \sigma_{1}, \ldots, \sigma_{n-p})=0 \text { for } i=1, \ldots, n-2,
$$

thus each $n$-tuple

$$
\left(K_{1}, \ldots, K_{n}\right)=(U_{i}, \underbrace{K, \ldots, K}_{p-1}, \sigma_{1}, \ldots, \sigma_{n-p})
$$

is degenerate, by (P7). Therefore, the following holds (see [5, Theorem 5.1.7]). There are a number $k \in\{1, \ldots, n\}$ and indices $1 \leq i_{1}<\cdots<i_{k} \leq n$ such that

$$
\operatorname{dim}\left(K_{i_{1}}+\cdots+K_{i_{k}}\right)<k
$$

Let $s \leq p-1$ be the number of indices among $i_{1}, \ldots, i_{k}$ which are from $\{2, \ldots, p\}$, and suppose that $s \geq 1$. Then either $k-s$ or $k-s-1$ of the indices are from $\{p+1, \ldots, n\}$. Since $\operatorname{dim} K=p$ and $\operatorname{lin} K$ and $\operatorname{span}\left\{\sigma_{1}, \ldots, \sigma_{n-p}\right\}$ are complementary subspaces, we get

$$
\operatorname{dim}\left(K_{i_{1}}+\cdots+K_{i_{k}}\right) \geq p+k-s-1 \geq k
$$

a contradiction. Hence, $s=0$. From (7) it then follows that $U_{i} \subset \operatorname{span}\left\{\sigma_{1}, \ldots, \sigma_{n-p}\right\}$, for $i=1, \ldots, n-2$. Therefore, $p=2$ and $\operatorname{span}\left\{U_{1}, \ldots, U_{n-2}\right\}=\operatorname{span}\left\{\sigma_{1}, \ldots, \sigma_{n-2}\right\}$. This space is the orthogonal complement of a two-dimensional subspace $Y$, which contains the convex body $K$ (by Firey's construction). We put

$$
T B:=K
$$

and have thus defined a map $T: \mathcal{K}_{(0)}(E) \rightarrow \mathcal{K}(Y)$. In the following lemma, we determine this map explicitly. For this, the previously introduced objects $U_{1}, \ldots, U_{n-1}, E, Y$ remain fixed, and we introduce the following notation. By $\pi_{Y}: \mathbb{R}^{n} \rightarrow Y$ we denote the orthogonal projection. We write $M={ }_{\operatorname{tr}} M^{\prime}\left(M \subset_{\operatorname{tr}} M^{\prime}\right)$ for convex bodies $M, M^{\prime}$ if and only if there exists a vector $t$ with $M=M^{\prime}+t$ (respectively, $M \subset M^{\prime}+t$ ). Further, we put $P:=U_{1}+\cdots+U_{n-2}$ and denote by $|P|$ the $(n-2)$-dimensional volume of $P$. (If $n=2$, we omit $P$ and replace $|P|$ by 1.)

Lemma 3. The map $T$ can be extended to an additive, continuous map on all of $\mathcal{K}(E)$. The extension satisfies

$$
T B=\operatorname{tr}_{\operatorname{tr}} b\left[\alpha \pi_{Y} B+(1-\alpha) \pi_{Y} B^{*}\right] \quad \text { for } B \in \mathcal{K}(E),
$$


with

$$
b=\frac{a|P|}{(n-1)(n-2) !},
$$

where $a$ is a positive constant depending only on $F$, and with a constant $\alpha \in[0,1]$, possibly depending also on $U_{1}, \ldots, U_{n-2}$ and $E$.

Proof. We need the following conclusion from Firey's [2] proof in Case 1. In our situation, $L=Y$, and the restriction of $\mu$ to $S^{n-1} \cap Y$ is the length measure $S_{1}(K, \cdot)$. For $u \in S^{n-1} \cap Y$, we have $h(A, u)=h\left(\pi_{Y} A, u\right)$, hence (6) reduces to

$$
\begin{aligned}
F\left(A, B, U_{1}, \ldots, U_{n-2}\right) & =\frac{1}{n} \int_{S^{n-1} \cap Y} h\left(\pi_{Y} A, u\right) S_{1}(T B, \mathrm{~d} u) \\
& =\frac{2}{n} v\left(\pi_{Y} A, T B\right),
\end{aligned}
$$

for $A \in \mathcal{K}^{n}$, where $v$ denotes the mixed volume in the two-dimensional space $Y$.

We make repeatedly use of the following fact. If $M, M^{\prime} \in \mathcal{K}(Y)$, then

$$
v(A, M) \leq v\left(A, M^{\prime}\right) \text { for all } A \in \mathcal{K}(Y) \text { implies } M \subset_{\operatorname{tr}} M^{\prime} .
$$

This result, which extends to higher dimensions but is used here only for $n=2$, is due to Weil [7].

Let $B_{1}, B_{2} \in \mathcal{K}_{(0)}(E)$. From the additivity of $F$ in its second argument and from (10) we get

$$
v\left(A, T\left(B_{1}+B_{2}\right)\right)=v\left(A, T B_{1}\right)+v\left(A, T B_{2}\right)=v\left(A, T B_{1}+T B_{2}\right)
$$

for all $A \in \mathcal{K}(Y)$ and hence $T\left(B_{1}+B_{2}\right)={ }_{\operatorname{tr}} T\left(B_{1}\right)+T\left(B_{2}\right)$. Since each body $T B$ has its Steiner point at the origin, it follows that

$$
T\left(B_{1}+B_{2}\right)=T B_{1}+T B_{2} .
$$

Thus, $T$ is additive.

In a similar way, it follows from the positive homogeneity of $F$ in its second argument that

$$
T(\lambda B)=\lambda T B
$$

for $\lambda \geq 0$.

Suppose that $B_{1} \subset_{\operatorname{tr}} B_{2}$. Since $F$ is increasing in its second argument, (10) gives

$$
v\left(A, T B_{1}\right) \leq v\left(A, T B_{2}\right) \quad \text { for all } A \in \mathcal{K}(Y),
$$

and we conclude that

$$
B_{1} \subset_{\operatorname{tr}} B_{2} \Rightarrow T B_{1} \subset_{\operatorname{tr}} T B_{2}
$$

We show that $T$ is continuous. Let $\left(B_{i}\right)_{i \in \mathbb{N}}$ be a sequence in $\mathcal{K}_{(0)}(E)$ converging to some convex body $B \in \mathcal{K}_{(0)}(E)$. There is a fixed convex body $C \in \mathcal{K}_{(0)}(E)$ containing all the $B_{i}$, hence $T B_{i} \subset T C$. Thus, the sequence $\left(T B_{i}\right)_{i \in \mathbb{N}}$ is bounded and hence has a 
convergent subsequence, say $T B_{i_{j}} \rightarrow M \in \mathcal{K}(Y)$ for $j \rightarrow \infty$. This convergence implies that $(n / 2) F\left(A, B_{i_{j}}, U_{1}, \ldots, U_{n-2}\right)=v\left(A, T B_{i_{j}}\right) \rightarrow v(A, M)$ for all $A \in \mathcal{K}(Y)$. From the continuity of $F$ we have $(n / 2) F\left(A, B_{i_{j}}, U_{1}, \ldots, U_{n-2}\right) \rightarrow(n / 2) F\left(A, B, U_{1}, \ldots, U_{n-2}\right)=$ $v(A, T B)$. The equality $v(A, M)=v(A, T B)$ for all $A \in \mathcal{K}(Y)$ yields $T B=\operatorname{tr} M$ and then $T B=M$, since both bodies have their Steiner point at the origin. Since every convergent subsequence of $\left(T B_{i}\right)_{i \in \mathbb{N}}$ converges to $T B$, the sequence itself converges to $T B$. This establishes the continuity of $T$ on $\mathcal{K}_{(0)}(E)$.

We extend $T$ to all of $\mathcal{K}(E)$. Let $S \in \mathcal{K}(E)$ be a segment or a singleton. Let $B_{i}, B \in \mathcal{K}_{(0)}(E)$ be such that $B_{i} \rightarrow S$. Then $B_{i}+B \rightarrow S+B$, hence $T B_{i}+T B=$ $T\left(B_{i}+B\right) \rightarrow T(S+B)$. From this it follows that the sequence $\left(T B_{i}\right)_{i \in \mathbb{N}}$ converges to some convex body $M$ and that $M+T B=T(S+B)$. Therefore, $M$ is uniquely determined, and we can define $T S:=M$. Then $T(S+B)=T S+T B$ for arbitrary $B \in \mathcal{K}_{(0)}(E)$. If $S_{1}, S_{2} \in \mathcal{K}(E)$, we choose $B \in \mathcal{K}_{(0)}(E)$ and have $T\left(S_{1}+S_{2}\right)+T(B)=$ $T\left(S_{1}+S_{2}+B\right)=T S_{1}+T\left(S_{2}+B\right)=T S_{1}+T S_{2}+T B$, hence $T\left(S_{1}+S_{2}\right)=T S_{1}+T S_{2}$. Thus, the extended mapping $T$ is additive. Similarly, if $B_{i} \rightarrow S$, then $B_{i}+B \rightarrow B+S$, hence $T\left(B_{i}+B\right) \rightarrow T(S+B)$ and thus $T B_{i}+T B \rightarrow T S+T B$, which implies $T B_{i} \rightarrow T B$. Hence, the extended map $T$ is continuous. By similar arguments, the other properties of $T$ (homogeneity, monotonicity) can be extended.

Let $C \in \mathcal{K}_{(0)}(E)$ be $o$-symmetric. For $A \in \mathcal{K}(E)$ it follows from Theorem 2 that

$$
F\left(A, C, U_{1}, \ldots, U_{n-2}\right)=a V\left(A, C, U_{1}, \ldots, U_{n-2}\right),
$$

where the constant $a$ is positive, since we have assumed that $F \not \equiv 0$. Recalling that $P=U_{1}+\cdots+U_{n-2}$, we have

$$
(n-2) ! V\left(A, C, U_{1}, \ldots, U_{n-2}\right)=V(A, C, P, \ldots, P),
$$

and formula $[5,(5.3 .23)]$ gives

$$
\left(\begin{array}{l}
n \\
2
\end{array}\right) V(A, C, P, \ldots, P)=|P| v\left(\pi_{Y} A, \pi_{Y} C\right) .
$$

Hence,

$$
F\left(A, C, U_{1}, \ldots, U_{n-2}\right)=\frac{1}{(n-2) !} \frac{1}{\left(\begin{array}{c}
n \\
2
\end{array}\right)}|P| v\left(\pi_{Y} A, \pi_{Y} C\right) .
$$

From (10) we have

$$
F\left(A, C, U_{1}, \ldots, U_{n-2}\right)=\frac{2}{n} v\left(\pi_{Y} A, T C\right) .
$$

Since, in the last two equations, $\pi_{Y} A$ can be any convex body in $Y$, we deduce from (11) that $T C={ }_{\operatorname{tr}} b \pi_{Y} C$, where $b$ is given by (9).

The body $C$ is centrally symmetric, hence also $T C$ is centrally symmetric (as follows from Firey's proof of Lemma 2). Since both sets have their centre at the origin, we conclude that $T C=b \pi_{Y} C$. Defining the linear map $\Lambda: E \rightarrow Y$ as the restriction of $b \pi_{Y}$ to $E$, we thus have

$$
T C=\Lambda C \quad \text { for } C \in \mathcal{K}(E) \text { with } C=-C .
$$


By continuity, this holds also if $\operatorname{dim} C<2$.

To study $T$ on nonsymmetric sets, we define $T^{\prime}: \mathcal{K}(Y) \rightarrow \mathcal{K}(Y)$ by $T^{\prime}(B):=$ $T\left(\Lambda^{-1} B\right)$ for $B \in \mathcal{K}(Y)$; then

$$
T^{\prime} C=C \quad \text { for } C \in \mathcal{K}(Y) \text { with } C=-C .
$$

We start with a triangle $\Delta \subset Y$. The set $\Delta+\Delta^{*}=: H\left(\right.$ recall that $\left.\Delta^{*}:=-\Delta\right)$ is a $o$-symmetric hexagon. It follows that

$$
T^{\prime} \Delta+T^{\prime} \Delta^{*}=T^{\prime}\left(\Delta+\Delta^{*}\right)=T^{\prime} H=H .
$$

Hence, $T^{\prime} \Delta$ and $T^{\prime} \Delta^{*}$ are summands of $H$. Let $S_{1}, S_{2}, S_{3}$ be the $o$-symmetric segments which are translates of the edges of $\Delta$. Since $\Delta$ and $\Delta^{*}$ both contain a translate of $S_{i}$, it follows from (12) that each of $T^{\prime} \Delta$ and $T^{\prime} \Delta^{*}$ contains a translate of $T^{\prime} S_{i}=S_{i}$, $i=1,2,3$.

The facts observed in the previous paragraph are invariant under affine transformations of $Y$. We may, therefore, introduce a scalar product $\langle\cdot, \cdot\rangle$ on $\mathbb{R}^{n}$ for which $\Delta$ becomes regular. Without loss of generality, we assume that $\Delta$ has vertices $o, u_{1}, u_{2}$, where $u_{1}, u_{2} \in Y$ are unit vectors. We put $u_{3}:=u_{2}-u_{1}$, then

$$
\left\langle u_{1}, u_{2}\right\rangle=\left\langle u_{3}, u_{2}\right\rangle=\frac{1}{2} .
$$

The edges of the hexagon $H$ are, say in clockwise order, translates of the segments $S_{1}, S_{2}, S_{3}, S_{1}, S_{2}, S_{3}$. Since $T^{\prime} \Delta$ is a summand of $H$, it has corresponding edges which are translates of $\alpha_{1} S_{1}, \alpha_{2} S_{2}, \alpha_{3} S_{3}, \alpha_{4} S_{1}, \alpha_{5} S_{2}, \alpha_{6} S_{3}$ with $0 \leq \alpha_{i} \leq 1$ (here we allow edges of length zero). The triangle $T^{\prime} \Delta^{*}$ has corresponding edges which are translates of $\left(1-\alpha_{1}\right) S_{1},\left(1-\alpha_{2}\right) S_{2},\left(1-\alpha_{3}\right) S_{3},\left(1-\alpha_{4}\right) S_{1},\left(1-\alpha_{5}\right) S_{2},\left(1-\alpha_{6}\right) S_{3}$.

Let $x, y$ be the endpoints of a longest segment parallel to $S_{2}$ contained in $T^{\prime} \Delta$. We may assume that $y-x=r u_{2}$ with $r>0$. Since $T^{\prime} \Delta$ contains a translate of $S_{2}$, we have $r \geq 1$. We may further assume, as is easy to see, that $x$ is a vertex of $T^{\prime} \Delta$. Then either

$$
y=x+\alpha_{1} u_{1}+\alpha_{2} u_{2}+\lambda \alpha_{3} u_{3}
$$

with $0 \leq \lambda \leq 1$, or

$$
y=x+\alpha_{1} u_{1}+\alpha_{2} u_{2}+\alpha_{3} u_{3}-\mu \alpha_{4} u_{1}
$$

with $0 \leq \mu \leq 1$. In the first case, we obtain

$$
1 \leq r=\left\langle y-x, u_{2}\right\rangle=\frac{1}{2} \alpha_{1}+\alpha_{2}+\frac{1}{2} \lambda \alpha_{3} \leq \frac{1}{2} \alpha_{1}+\alpha_{2}+\frac{1}{2} \alpha_{3},
$$

and in the second case

$$
1 \leq r=\left\langle y-x, u_{2}\right\rangle=\frac{1}{2} \alpha_{1}+\alpha_{2}+\frac{1}{2} \alpha_{3}-\frac{1}{2} \mu \alpha_{4} \leq \frac{1}{2} \alpha_{1}+\alpha_{2}+\frac{1}{2} \alpha_{3} .
$$

In a similar way, considering the triangle $T^{\prime} \Delta^{*}$, we obtain

$$
1 \leq \frac{1}{2}\left(1-\alpha_{1}\right)+\left(1-\alpha_{2}\right)+\frac{1}{2}\left(1-\alpha_{3}\right) .
$$


Adding (14), respectively (15), to (16), we see that equality must hold in (14), respectively (15), which means that

$$
y-x=\alpha_{1} u_{1}+\alpha_{2} u_{2}+\alpha_{3} u_{3}
$$

For a unit vector $v \in Y$ orthogonal to $u_{2}$ we have $\left\langle u_{3}, v\right\rangle=\left\langle u_{2}-u_{1}, v\right\rangle=-\left\langle u_{1}, v\right\rangle \neq 0$, hence (17) gives $\alpha_{1}=\alpha_{3}$. In the same way (going 'the other way round' from $x$ to $y$ ) we obtain that $\alpha_{4}=\alpha_{6}$.

Applying the same argument to $S_{1}$ or $S_{3}$, we deduce that $\alpha_{1}=\alpha_{3}=\alpha_{5}=: \alpha$ and $\alpha_{2}=\alpha_{4}=\alpha_{6}=: \beta$. Since (14) holds with equality, we have $\alpha+\beta=1$. Thus, we have obtained that

$$
T^{\prime} \Delta=\operatorname{tr} \alpha(\Delta) \Delta+(1-\alpha(\Delta)) \Delta^{*},
$$

where we have written $\alpha(\Delta)$ to point out a possible dependence on $\Delta$. It remains to show that there is, in fact, no such dependence.

For this, let $G$ be a line and $z$ a point in $Y$ with $z \notin G$. Let $u, v, w$ be different points on $G$, in this order, and let $\Delta_{1}:=\operatorname{conv}\{z, u, v\}, \Delta_{2}:=\operatorname{conv}\{z, v, w\}, \Delta:=$ $\operatorname{conv}\{z, u, w\}, S:=\operatorname{conv}\{z, v\}$ and write $\alpha\left(\Delta_{i}\right)=: \alpha_{i}$ for $i=1,2$ and $\alpha(\Delta)=: \alpha$. Then $\Delta+S=\Delta_{1}+\Delta_{2}$ and therefore

$$
\begin{aligned}
& \alpha\left(\Delta_{1}+\Delta_{2}\right)+(1-\alpha)\left(\Delta_{1}+\Delta_{2}\right)^{*} \\
& =\alpha(\Delta+S)+(1-\alpha)(\Delta+S)^{*} \\
& =\alpha \Delta+(1-\alpha) \Delta^{*}+S={ }_{\operatorname{tr}} T^{\prime} \Delta+S=T^{\prime} \Delta+T^{\prime} S \\
& =T^{\prime}(\Delta+S)=T^{\prime}\left(\Delta_{1}+\Delta_{2}\right)=T^{\prime} \Delta_{1}+T^{\prime} \Delta_{2} \\
& =\operatorname{tr} \alpha_{1} \Delta_{1}+\left(1-\alpha_{1}\right) \Delta_{1}^{*}+\alpha_{2} \Delta_{2}+\left(1-\alpha_{2}\right) \Delta_{2}^{*} .
\end{aligned}
$$

Let $\xi \in Y$ denote the outer unit normal vector of the edge $\operatorname{conv}\{z, u\}$ of the triangle $\Delta_{1}$. The support sets of the polygons (18) and (19) with outer unit normal vector $\xi$ must have the same length, hence $\alpha=\alpha_{1}$ (note that none of the triangles $\Delta_{2}, \Delta_{1}^{*}, \Delta_{2}^{*}$ has an edge with outer normal vector $\xi$ ). Therefore, for $u, w \in G$ the value $\alpha(\Delta)$ for $\Delta:=\operatorname{conv}\{z, u, w\}$ depends only on $u$. For the same reason, it depends only on $w$. Hence, it is a constant for $u, w \in G$. Since $T^{\prime}$ is positively homogeneous, the value $\alpha(\Delta)$ is constant for all triangles $\Delta$ with an edge on $G$. Taking a line $G^{\prime}$ intersecting $G$ and a triangle with edges on $G^{\prime}$ and $G$, we see that the value is also independent of the line. Therefore, $T^{\prime} \Delta=\operatorname{tr}_{\operatorname{tr}} \alpha \Delta+(1-\alpha) \Delta^{*}$ with a fixed $\alpha \in[0,1]$, for all triangles $\Delta$. Since every convex polygon $P$ is a sum of triangles and segments, we get $T^{\prime} P=\operatorname{tr} \alpha P+(1-\alpha) P^{*}$ and then, by approximation and continuity, $T^{\prime} B={ }_{\operatorname{tr}} \alpha B+(1-\alpha) B^{*}$ for all $B \in \mathcal{K}(Y)$.

Recalling that $T^{\prime}(B)=T\left(\Lambda^{-1} B\right)$ for $B \in \mathcal{K}(Y)$ and that $\Lambda=\left.b \pi_{Y}\right|_{E}$, we arrive at (8). This finishes the proof of Lemma 3.

Now we complete the proofs of Theorem 3 and Theorem 4 separately. 
Proof of Theorem 3. If $n=2$, no segments $U_{1}, \ldots, U_{n-2}$ appear, and the previous proof and result hold with $E=Y=\mathbb{R}^{2}$ and $|P|=1$. In view of (9), equation (8) simplifies to

$$
T B=\operatorname{tr} a\left[\alpha B+(1-\alpha) B^{*}\right] \quad \text { for } B \in \mathcal{K}^{2} .
$$

Hence, (10) gives

$$
F(A, B)=a V\left(A, \alpha B+(1-\alpha) B^{*}\right)
$$

for $A, B \in \mathcal{K}^{2}$. This completes the proof of Theorem 3 .

Proof of Theorem 4. Now we assume that $n \geq 3$ and that $F$ satisfies also condition (P6). From (8) and (10) we have

$$
F\left(A, B, U_{1}, \ldots, U_{n-2}\right)=\frac{2}{n} b v\left(\pi_{Y} A, \pi_{Y}\left[\alpha B+(1-\alpha) B^{*}\right]\right)
$$

for $A \in \mathcal{K}^{n}$ and $B \in \mathcal{K}(E)$.

So far, the linearly independent segments $U_{1}, \ldots, U_{n-2}$ and the two-dimensional linear subspace $E$, complementary to $\operatorname{span}\left\{U_{1}, \ldots, U_{n-2}\right\}$, were fixed. The constant $\alpha$ in (20) may depend on this data, therefore we write $\alpha=\alpha\left(U_{1}, \ldots, U_{n-2}, E\right)$. To show the independence, we first fix $U_{1}, \ldots, U_{n-2}$ and consider two two-dimensional subspaces $E_{1}, E_{2}$, both complementary to $\operatorname{span}\left\{U_{1}, \ldots, U_{n-2}\right\}$. Write $\alpha\left(U_{1}, \ldots, U_{n-2}, E_{i}\right)=: \alpha_{i}$. Let $B \in \mathcal{K}(Y)$ and $B_{i} \in \mathcal{K}\left(E_{i}\right)$ be convex bodies such that $\pi_{Y} B_{i}=B, i=1,2$. It follows from Proposition 3 (modified for the appropriate variables) that

$$
F\left(A, B_{1}, U_{1}, \ldots, U_{n-2}\right)=F\left(A, B_{2}, U_{1}, \ldots, U_{n-2}\right),
$$

hence (20), together with $\pi_{Y} B_{i}=B$, gives

$$
v\left(\pi_{Y} A, \alpha_{1} B+\left(1-\alpha_{1}\right) B^{*}\right)=v\left(\pi_{Y} A, \alpha_{2} B+\left(1-\alpha_{2}\right) B^{*}\right) .
$$

Since this holds, in particular, for all $A \in \mathcal{K}(Y)$, we deduce from (11) that

$$
\alpha_{1} B+\left(1-\alpha_{1}\right) B^{*}={ }_{\operatorname{tr}} \alpha_{2} B+\left(1-\alpha_{2}\right) B^{*} .
$$

Choosing for $B$ a triangle in $Y$, we conclude that $\alpha_{1}=\alpha_{2}$. Thus, $\alpha\left(U_{1}, \ldots, U_{n-2}, E\right)$ does not depend on $E$, and we denote it by $\alpha\left(U_{1}, \ldots, U_{n-2}\right)$. From (20) and (9) we can deduce that $\alpha\left(U_{1}, \ldots, U_{n-2}\right)$ does not change if, say, $U_{1}$ is dilated by a positive factor.

Now let $\left(U_{1}^{\prime}, \ldots, U_{n-2}^{\prime}\right)$ be a second $(n-2)$-tuple of centred segments with linearly independent directions and such that

$$
\operatorname{span}\left\{U_{1}, \ldots, U_{n-2}\right\}=\operatorname{span}\left\{U_{1}^{\prime}, \ldots, U_{n-2}^{\prime}\right\}=Y^{\perp} .
$$

We write $P^{\prime}=U_{1}^{\prime}+\cdots+U_{n-2}^{\prime}$ and $\alpha\left(U_{1}, \ldots, U_{n-2}\right)=\alpha, \alpha\left(U_{1}^{\prime}, \ldots, U_{n-2}^{\prime}\right)=\alpha^{\prime}$. Since $\alpha\left(U_{1}, \ldots, U_{n-2}\right)$ does not change under dilatation of one of its arguments, we can assume that $|P|=\left|P^{\prime}\right|$. Then there exists a linear map $\varphi \in \operatorname{SL}(n)$ with $\varphi U_{i}=U_{i}^{\prime}$ for 
$i=1, \ldots, n-2$ and $\varphi y=y$ for $y \in Y$. Now we use property (P6). For $A, B \in \mathcal{K}(Y)$ we obtain from (20) that

$$
\begin{aligned}
& \frac{2}{n} b v\left(A, \alpha B+(1-\alpha) B^{*}\right) \\
& =F\left(A, B, U_{1}, \ldots, U_{n-2}\right)=F\left(\varphi A, \varphi B, \varphi U_{1}, \ldots, \varphi U_{n-2}\right)=F\left(A, B, U_{1}^{\prime}, \ldots, U_{n-2}^{\prime}\right) \\
& =\frac{2}{n} b v\left(A, \alpha^{\prime} B+\left(1-\alpha^{\prime}\right) B^{*}\right) .
\end{aligned}
$$

By the argument used above, we can conclude that $\alpha=\alpha^{\prime}$. This shows that $\alpha\left(U_{1}, \ldots, U_{n-2}\right)$ depends, in fact, only on $Y=\operatorname{span}\left\{U_{1}, \ldots, U_{n-2}\right\}^{\perp}$, and we denote it by $\alpha(Y)$.

Let $Y^{\prime}$ be a second two-dimensional linear subspace. There exists a rotation $\rho \in$ $\mathrm{SO}(n)$ with $Y^{\prime}=\rho Y$. For $A, B \in \mathcal{K}(Y)$ we get

$$
\begin{aligned}
& \frac{2}{n} b v\left(A, \alpha(Y) B+(1-\alpha(Y)) B^{*}\right) \\
& =F\left(A, B, U_{1}, \ldots, U_{n-2}\right)=F\left(\rho A, \rho B, \rho U_{1}, \ldots, \rho U_{n-2}\right) \\
& =\frac{2}{n} b v\left(\rho A, \alpha\left(Y^{\prime}\right) \rho B+\left(1-\alpha\left(Y^{\prime}\right)\right) \rho B^{*}\right) \\
& =\frac{2}{n} b v\left(A, \alpha\left(Y^{\prime}\right) B+\left(1-\alpha\left(Y^{\prime}\right)\right) B^{*}\right),
\end{aligned}
$$

by the rigid motion invariance of the mixed volume. As above, we conclude that $\alpha(Y)=\alpha\left(Y^{\prime}\right)$.

We have shown that the constant $\alpha$ in (20) is independent of $U_{1}, \ldots, U_{n-2}$ and the two-dimensional subspace in which $B$ lies. We can write (20) in the form

$$
F\left(A, B, U_{1}, \ldots, U_{n-2}\right)=a V\left(A, \alpha B+(1-\alpha) B^{*}, U_{1}, \ldots, U_{n-2}\right),
$$

valid for $A \in \mathcal{K}^{n}$ and two-dimensional convex bodies $B \in \mathcal{K}^{n}$. By continuity, the assumption on the linear independenc of the directions of $U_{1}, \ldots, U_{n-2}$ is no longer required. That $(21)$ holds, in fact, for general convex bodies $B \in \mathcal{K}^{n}$, can be shown by a similar extension procedure as in Section 2, replacing generalized zonoids by generalized triangle bodies. A convex body $K \in \mathcal{K}^{n}$ is called a triangle body if it can be approximated by finite sums of triangles, and it is called a generalized triangle body if there exist triangle bodies $T_{1}, T_{2}$ such that $K+T_{1}=T_{2}$. By [6, Lemma 2], the set of generalized triangle bodies is dense in $\mathcal{K}^{n}$. Therefore, the kind of argument from the proof of Theorem 2 can be used to extend (21) from two-dimensional convex bodies $B$ to general convex bodies.

As in Section 2, each argument $U_{i}$ can be replaced by a centrally symmetric convex body, thus we get

$$
F\left(A, B, C_{1}, \ldots, C_{n-2}\right)=a V\left(A, \alpha B+(1-\alpha) B^{*}, C_{1}, \ldots, C_{n-2}\right)
$$

for $A, B \in \mathcal{K}^{n}$ and $C_{1}, \ldots, C_{n-2} \in \mathcal{K}_{s}^{n}$. This finishes the proof of Theorem 4. 


\section{Proof of Theorem 5}

Let $n=2$, and let $F$ have the properties of Theorem 5 . By Lemma $1, F$ is non-negative, in each variable translation invariant, continuous and positively homogeneous, and it vanishes if one of its arguments is a singleton.

In the following, all segments are again nondegenerate. Let $S$ be a segment. By $\left(\mathrm{P} 5^{\prime}\right)$, there exists a segment $\bar{S}$ with $F(S, \bar{S})=0$, and every segment parallel to $\bar{S}$ has the same property. We make $\bar{S}$ unique by demanding that it has centre $o$ and length 1 , with respect to a given Euclidean metric. Then $\left(\mathrm{P}^{\prime}\right)$ implies for segments $S_{1}$ that

$$
F\left(S, S_{1}\right)=0 \Leftrightarrow S_{1} \| \bar{S}
$$

Similarly, to each segment $S$ there exists a unique centred segment $\tilde{S}$ of unit length such that

$$
F\left(S_{1}, S\right)=0 \Leftrightarrow S_{1} \| \tilde{S}
$$

We modify the proof of Theorem 3. Let $B \in \mathcal{K}_{(0)}^{2}$, the set of two-dimensional convex bodies in $\mathbb{R}^{2}$. The function $A \mapsto F(A, B), A \in \mathcal{K}^{2}$, is not identically zero, since otherwise $F\left(S, S_{1}\right)=0$ for an arbitrary segment $S$ and all segments $S_{1} \subset B$, which contradicts (22). Now we can apply Lemma 2 . Its case 2 cannot occur, for the same reason as just explained. Hence, there is a unique convex body $K \in \mathcal{K}_{(0)}^{2}$, with Steiner point $o$, that satisfies $F(A, B)=V(A, K)$ for all $A \in \mathcal{K}^{2}$, and we can define a map $T: \mathcal{K}_{(0)}^{2} \rightarrow \mathcal{K}_{(0)}^{2}$ by $T B:=K$. Precisely as in Section 3 it follows that $T$ is additive, positively homogeneous, increasing up to translations, continuous, and can be extended to a mapping $T: \mathcal{K}^{2} \rightarrow \mathcal{K}^{2}$ under preservation of all these properties. In particular, we have

$$
F(A, B)=V(A, T B) \quad \text { for all } A, B \in \mathcal{K}^{2} .
$$

Let $x \in \mathbb{R}^{2}$ (and recall that we identify $\{x\}$ with $x$ ). Since $F(A, x)=0$ for all $A \in \mathcal{K}^{2}$, we obtain $V(A, T x)=0$ for all $A \in \mathcal{K}^{2}$. This implies

$$
T x=o .
$$

We want to use the properties of $T$ to show that it is induced by a linear mapping. First we assert that under the mapping $T$ the following holds.

(a) Only singletons are mapped to singletons.

(b) Segments are mapped to segments.

(c) Segments of different directions are mapped to segments of different directions.

Proof of (a): If $T B$ is a singleton, then (24) yields $F(A, B)=V(A, T B)=0$ for all $A \in \mathcal{K}^{2}$. If $B$ is not a singleton, it contains a segment $S$, and we would have $F\left(S_{1}, S\right)=0$ for all segments $S_{1}$, which contradicts (23). This contradiction shows that $B$ is a singleton.

Proof of (b): Assume that $S$ is a segment and $T S$ is not a segment. By (a), TS is not a singleton, hence $\operatorname{dim} T S=2$. It follows that $0=F(\tilde{S}, S)=V(\tilde{S}, T S) \neq 0$, since $\tilde{S}$ is not a singleton, a contradiction. 
Proof of (c): Let $S_{1}, S_{2}$ be segments of different directions. As just proved, $T S_{1}$ and $T S_{2}$ are segments. Assume, to the contrary, that $T S_{1} \| T S_{2}$. Then (24) gives $F\left(T S_{1}, S_{2}\right)=V\left(T S_{1}, T S_{2}\right)=0$, and $(23)$ implies that $T S_{1} \| \tilde{S}_{2}$. This gives $F\left(\tilde{S}_{2}, S_{1}\right)=$ $V\left(\tilde{S}_{2}, T S_{1}\right)=0$ and hence $\tilde{S}_{2} \| \tilde{S}_{1}$. Therefore, $F\left(\tilde{S}_{1}, S_{2}\right)=0$. Since also $F\left(\tilde{S}_{1}, S_{1}\right)=0$ by the definition of $\tilde{S}_{1}$, now (22) (applied to $\tilde{S}_{1}$ instead of $S$ ) yields that $S_{1} \| S_{2}$, a contradiction. This completes the proof of (c).

For $x \in \mathbb{R}^{2} \backslash\{o\}$ we denote by $S_{x}$ the segment with endpoints $\pm x$. The image $T S_{x}$ is a segment with centre $o$, hence of the form $S_{y}$ with $y \in \mathbb{R}^{2} \backslash\{o\}$. For $\lambda \geq 0$ we have

$$
T S_{\lambda x}=T\left(|\lambda| S_{x}\right)=|\lambda| T S_{x}=|\lambda| S_{y}=S_{\lambda y}
$$

We fix two linearly independent vectors $a, b \in \mathbb{R}^{2}$. Then $T S_{a}=S_{u}, T S_{b}=S_{v}$ with linearly independent vectors $u, v \in \mathbb{R}^{2}$. By the homogeneity of $T$, also $T S_{\lambda a}=S_{\lambda u}$, $T S_{\lambda b}=S_{\lambda v}$ for $\lambda>0$.

We consider the diagonals of the parallelogram $S_{a}+S_{b}$. We have

$$
S_{a+b} \subset S_{a}+S_{b}, \quad S_{a-b} \subset S_{a}+S_{b}
$$

and

$$
S_{2 a} \subset S_{a+b}+S_{a-b}, \quad S_{2 b} \subset S_{a+b}+S_{a-b} .
$$

From (12) and $o$-symmetry we obtain that

$$
T S_{a+b} \subset S_{u}+S_{v}, \quad T S_{a-b} \subset S_{u}+S_{v}
$$

and

$$
2 S_{u} \subset T S_{a+b}+T S_{a-b}, \quad 2 S_{v} \subset T S_{a+b}+T S_{a-b} .
$$

We have $T S_{a+b}=S_{x}$ and $T S_{a-b}=S_{y}$ with linearly independent vectors $x, y \in \mathbb{R}^{2}$, say

$$
x=\lambda u+\mu v, \quad y=\gamma u+\delta v .
$$

Then (25) implies that $|\lambda|,|\mu|,|\gamma|,|\delta| \leq 1$. From

$$
u=\frac{\delta}{D} x-\frac{\mu}{D} y, \quad v=-\frac{\gamma}{D} x+\frac{\lambda}{D} y, \quad D=\lambda \delta-\mu \gamma
$$

and (26) we obtain $|\lambda|,|\mu|,|\gamma|,|\delta| \leq|D| / 2$. This gives $|D| \leq|\lambda \delta|+|\mu \gamma| \leq D^{2} / 2$, hence $|D| \geq 2$. On the other hand, $|D| \leq|\lambda \delta|+|\mu \gamma| \leq 2$. We conclude that $|\lambda|,|\mu|,|\gamma|,|\delta|=1$. This means that either $T S_{a+b}=S_{u+v}, T S_{a-b}=S_{u-v}$ or $T S_{a+b}=S_{u-v}, T S_{a-b}=S_{u+v}$. Replacing $v$ by $-v$, if necessary, we can assume that $T S_{a+b}=S_{u+v}$ and $T S_{a-b}=S_{u-v}$.

Let $\lambda, \mu>0$. Since $T S_{\lambda a}=S_{\lambda u}, T S_{\mu v}=S_{\mu b}$, the same argument as above shows that either $T S_{\lambda a+\mu b}=S_{\lambda u+\mu v}, T S_{\lambda a-\mu b}=S_{\lambda u-\mu v}$ or $T S_{\lambda a+\mu b}=S_{\lambda u-\mu v}, T S_{\lambda a-\mu b}=$ $S_{\lambda u+\mu v}$. Since for $\lambda, \mu=1$ the first case occurs, it follows from the continuity of $T$ that the first case occurs for all $\lambda, \mu>0$. Now we define a (nondegenerate) linear map $\varphi: \mathbb{R}^{2} \rightarrow \mathbb{R}^{2}$ by

$$
\varphi(\lambda a+\mu b):=\lambda u+\mu v
$$


for $\lambda, \mu \in \mathbb{R}$. Then we have $T S=\varphi S$ for every centred segment $S \subset \mathbb{R}^{2}$ and hence

$$
T C=\varphi C \quad \text { for } C \in \mathcal{K}^{2} \text { with } C=-C .
$$

This equation corresponds to equation (13) in Section 3, with $E$ replaced by $\mathbb{R}^{2}$ and $\Lambda$ replaced by $\varphi$. We continue the proof verbally as there, to end up with an equation corresponding to (8), namely

$$
T B=\operatorname{tr} \varphi\left[\alpha B+(1-\alpha) B^{*}\right] \quad \text { for } B \in \mathcal{K}^{2},
$$

with $\alpha \in[0,1]$. Together with (24) this completes the proof of Theorem 5 .

\section{References}

[1] J. Aczél, Functional Equations and their Applications. Academic Press, New York 1966.

[2] W.J. Firey, A functional characterization of certain mixed volumes. Israel J. Math. 24 (1976), 274-281.

[3] D. Klain, A short proof of Hadwiger's characterization theorem. Mathematika 42 (1995), 329-339.

[4] K. Przesławski, Integral representations of mixed volumes and mixed moment tensors. Rend. Circ. Mat. Palermo (2) Suppl. 50 (1997), 299-314.

[5] R. Schneider, Convex Bodies: the Brunn-Minkowski Theory. Cambridge University Press, Cambridge 1993.

[6] R. Schneider, Simple valuations on convex bodies. Mathematika 43 (1996), 32-39.

[7] W. Weil, Decomposition of convex bodies. Mathematika 21 (1974), 19-25.

[8] W. Weil, Das gemischte Volumen als Distribution. Manuscripta Math. 36 (1981), $1-18$.

V.D. Milman, School of Mathematical Sciences, Tel Aviv University, Ramat Aviv, Tel Aviv 69978, Israel

E-mail: milman@post.tau.ac.il

R. Schneider, Mathematisches Institut, Albert-Ludwigs-Universität, Eckerstr. 1, 79104 Freiburg i. Br., Germany

E-mail: rolf.schneider@math.uni-freiburg.de 